\title{
Real causes of apparent abnormal results in heavy ion reactions
}

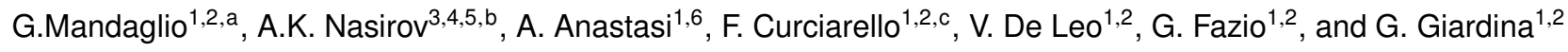 \\ ${ }^{1}$ Dipartimento di Fisica e di Scienze della Terra dell'Università di Messina, Salita Sperone 31, 98166, Messina, Italy \\ ${ }^{2}$ Istituto Nazionale di Fisica Nucleare, Sezione di Catania, Catania, Italy \\ ${ }^{3}$ Joint Institute for Nuclear Research, Joliot-Curie 6, 141980 Dubna, Russia \\ ${ }^{4}$ Institute of Nuclear Physics, Ulugbek, 100214, Tashkent, Uzbekistan \\ ${ }^{5}$ Kyungpook National University, 702-701, Daegu, Republic Korea \\ ${ }^{6}$ Istituto Nazionale di Fisica Nucleare, Laboratori Nazionali di Frascati, Italy
}

\begin{abstract}
We study the effect of the static characteristics of nuclei and dynamics of the nucleus-nucleus interaction in the capture stage of reaction, in the competition between quasifission and complete fusion processes, as well as the angular momentum dependence of the competition between fission and evaporation processes along the de-excitation cascade of the compound nucleus. The results calculated for the mass-asymmetric and less mass-asymmetric reactions in the entrance channel are analyzed in order to investigate the role of the dynamical effects on the yields of the evaporation residue nuclei. We also discuss about uncertainties at the extraction of such relevant physical quantities as $\Gamma_{n} / \Gamma_{\text {tot }}$ ratio or also excitation functions from the experimental results due to the not always realistic assumptions in the treatment and analysis of the detected events. This procedure can lead to large ambiguity when the complete fusion process is strongly hindered or when the fast fission contribution is large. We emphasize that a refined multiparameter model of the reaction dynamics as well as a more detailed and checked data analysis are strongly needed in heavy-ion collisions.
\end{abstract}

\section{Introduction}

The possibility of understanding the role of the shape, structure and relative motion of the colliding nuclei at the near Coulomb barrier energies in formation of the observed products can be ascertained by the comparison of the experimental data obtained from the reactions with different projectile- and target-nucleus leading to the formation of the same compound nucleus $(\mathrm{CN})$ (see for example Refs. [1-7]). In all of these works, the results of the comparison led to the same conclusions: the evaporation residue(ER) cross sections of the same heated and rotating $\mathrm{CN}$ are different even at the same value of the excitation energy $E_{\mathrm{CN}}^{*}$. The main conclusion of the authors in interpretations of the observed difference between ER cross sections is an appearance of the hindrance to complete fusion in the stage of the $\mathrm{CN}$ formation. The extent of the hindrance is determined by the peculiarities of the potential energy surface [refs. 3- 7] which contains shell effects of the intrinsic structure of interacting nuclei that change nature during the lifetime and evolution of the dinuclear system(DNS) formed at capture of the projectile by the target. The hindrance to complete fusion is related with the increase of the quasifission events (decay of DNS into two fragments) which are in competition with the complete fusion events. In many studies, the appearance of the quasi-

\footnotetext{
ae-mail: gmandaglio@unime.it

be-mail: nasirov@jinr.ru

ce-mail: fcurciarello@unime.it
}

fission products showed that their yields and mass distributions are determined by the mass (charge) asymmetry of the entrance channels and peculiarities of the potential energy surface.

The frequent trend observed in the total ER cross sections obtained by many reactions with different mass asymmetry in the entrance channel was that the total ER yield produced by a mass asymmetric reaction is larger than the one of an almost mass symmetric reaction, leading to the same $\mathrm{CN}$ or also to an heavier $\mathrm{CN}$; however, as happens several times in research, are the curious results that seem not to be easily understood that induce researchers to be particularly attentive and capable in the necessary improvement of theoretical models and/or of the experimental procedures and analysis of the data, in order to be able to achieve a proper understanding of the reaction processes that determine the observed results. To this end, in the present paper we discuss about the study done on various nuclear reactions, by comparing the theoretical results with the available experimental ones present in literature.

Therefore, we choose the following cases: four reactions from the mass asymmetric ${ }^{16} \mathrm{O}+{ }^{204} \mathrm{~Pb}$ and ${ }^{40} \mathrm{Ar}+{ }^{180} \mathrm{Hf}$ reactions to the almost mass symmetric ${ }^{82} \mathrm{Se}+{ }^{138} \mathrm{Ba}$ and ${ }^{124} \mathrm{Sn}+{ }^{96} \mathrm{Zr}$ reactions, all leading to the ${ }^{220} \mathrm{Th} \mathrm{CN}$; the two different mass asymmetric ${ }^{26} \mathrm{Mg}+{ }^{248} \mathrm{Cm}$ and ${ }^{36} \mathrm{~S}+{ }^{238} \mathrm{U}$ reactions leading to the superheavy hassium ${ }^{274} \mathrm{Hs} \mathrm{CN}$; the ${ }^{48} \mathrm{Ca}$ induced reactions on two different ${ }^{154,144} \mathrm{Sm}$ targets leading to different neutron rich isotopes of lead, ${ }^{202} \mathrm{~Pb}$ and 
${ }^{192} \mathrm{~Pb}$, respectively; the reactions with very close induced ${ }^{26,25} \mathrm{Mg}$ beams on the same ${ }^{248} \mathrm{Cm}$ target leading to very close superheavy hassium ${ }^{274,273} \mathrm{Hs}$ CNs. We chose a variegated sample of reactions in order to observe and study the dynamic effects of very different entrance channels on the compound nuclei and reaction product formations.

\section{Results and Discussion}

The study of heavy ion collisions at near Coulomb barrier energies is based on the calculations of the incoming path of the projectile on the target and finding capture probability taking into account the possibility of interaction with different orientation angles of the axial symmetry axis of the formed nuclei [refs. 7-9]. Also the surface vibration of the non-deformed nuclei is taken into account. The capture events of the projectile nuclei by the target nuclei are characterized by the full relative momentum transfer with strong energy dissipation. It is necessary that the reactant nuclei overcome the Coulomb barrier and the decrease of their relative kinetic energy due to dissipation by friction forces can lead to the projectile-like and the targetlike nuclei to be trapped in the pocket of potential forming a DNS with a residual relative kinetic energy lower than the depth of such a potential well [refs. 6,7,10]. It depends on the values of the beam energy and orbital angular momentum, the size of the potential well and intensity of the friction forces that cause dissipation of the kinetic energy of the relative motions to internal energy of two nuclei. So, the trapping of the collision path into the well is a capture process. The lifetime of the excited DNS and then the relative stability of DNS against decay into two fragments is determined by the depth of the potential well which is called quasifission $\operatorname{barrier}\left(B_{\mathrm{qf}}\right)$. Only part of the paths of the DNS evolution and surviving against decay along the relative distance lead to the deformed complete fusion of nuclear system, and consequently to the statistically equilibrated shape of $\mathrm{CN}$. The fusion probability $P_{\mathrm{CN}}(E, l)=\sigma_{\text {fus }}(E, \ell) / \sigma_{\text {cap }}(E, \ell)$ is used to take into account the competition between the complete fusion and decay of DNS into two fragments (quasifission) to calculate complete fusion cross section [refs. 6,7]:

$$
\sigma_{f u s}(E)=\Sigma_{\ell=0}^{\ell_{d}(E)}(2 \ell+1) \sigma_{c a p}(E, \ell) P_{C N}(E, \ell) .
$$

The decay of DNS without reaching the equilibrated shape of the $\mathrm{CN}$ formation $[7,10,11]$ is called quasifission process and its cross section is obtained by the following expression

$$
\sigma_{q f i s}(E)=\Sigma_{\ell=0}^{\ell_{d}(E)}(2 \ell+1) \sigma_{c a p}(E, \ell)\left(1-P_{C N}(E, \ell)\right) .
$$

Evaporation residue cross sections are calculated by light particle emission processes along the excitation cascade of the compound nucleus surviving fission at each step of the cascade by the expression

$$
\sigma_{E R}^{x}\left(E_{x}^{*}\right)=\Sigma_{\ell=0}^{\ell_{d}}(2 \ell+1) \sigma_{E R}^{x}\left(E_{x}^{*}, \ell\right) .
$$

where $E_{x}^{*}$ is the energy of the intermediate excited nucleus at each step $x$ along the de-excitation cascade, and

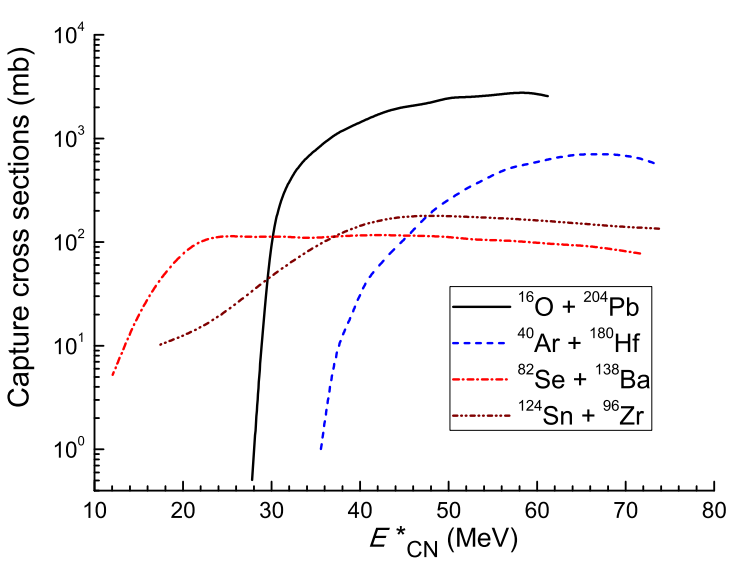

Figure 1. (Color online) Comparison of the capture excitation functions calculated for the ${ }^{16} \mathrm{O}+{ }^{204} \mathrm{~Pb}$ (solid line), ${ }^{40} \mathrm{Ar}+{ }^{180} \mathrm{Hf}$ (dashed), ${ }^{82} \mathrm{Se}+{ }^{138} \mathrm{Ba}$ (dot-dashed), and ${ }^{124} \mathrm{Sn}+{ }^{96} \mathrm{Zr}$ (dot-dot dashed) reactions.

$\sigma_{E R}^{x}\left(E_{x}^{*}, \ell\right)$ is the partial cross section of ER formation obtained by formula

$$
\left.\sigma_{E R}^{x}\left(E_{x}^{*}, \ell\right)=\sigma_{E R}^{x}\left(E_{x-1}^{*}, \ell\right) W_{\text {sur }}^{x}\left(E_{x}^{*}, \ell\right)\right) .
$$

In this last formula, $\sigma_{E R}^{x}\left(E_{x-1}^{*}, \ell\right)$ is the partial cross section of the intermediate nucleus formation at the $(x-1)$ th step, and $W_{\text {sur }}^{x}$ is the survival probability of the $x t h$ intermediate nucleus against fission along the de-excitation cascade of $\mathrm{CN}$. Due to the dependence of the fission barrier on the angular momentum $l$, the survival probability $W_{\text {sur }}\left(E_{\mathrm{CN}}^{*}, \ell\right)$ depends on $l$. The damping of the shell corrections determining the fission barrier is taken into account as in ref. [12].

The details of calculation of the capture, fusion and ER cross sections can be found in ref. $[6,7,13]$.

\subsection{Very different reactions leading to the same ${ }^{220}$ Th CN}

In Figs. 1, 2, and 3 we present and compare the capture, fusion, and evaporation residue excitation functions vs $E_{\mathrm{CN}}^{*}$, respectively, for the ${ }^{16} \mathrm{O}+{ }^{204} \mathrm{~Pb}$ (very mass asymmetric), ${ }^{40} \mathrm{Ar}+{ }^{180} \mathrm{Hf}$ (mass asymmetric), ${ }^{82} \mathrm{Se}+{ }^{138} \mathrm{Ba}$ (almost mass symmetric), and ${ }^{124} \mathrm{Sn}+{ }^{96} \mathrm{Zr}$ (more mass symmetric) reactions.

Hereafter, the $\mathrm{CN}$ excitation energy $E_{\mathrm{CN}}^{*}$ is used instead of the collision energy in the center-of-mass system $E_{\text {c.m. }}$ for the convenience of comparison of the reactions having large difference in the Coulomb barrier energies. The threshold values of $E_{\mathrm{CN}}^{*}$ for the capture excitation functions is determined by the Coulomb barriers of the entrance channel and by the reaction $Q_{\mathrm{gg}}$-value:

$E_{\mathrm{CN}}^{*}\left(Z=Z_{1}\right)=E_{\mathrm{c} . \mathrm{m} .}^{(\mathrm{min})}(Z)+Q_{\mathrm{gg}}(Z)=V_{B}^{(\mathrm{Coul})}(Z)+Q_{\mathrm{gg}}(Z)$.

$Q_{\mathrm{gg}}$ depends on the combinations of nuclei in the entrance channel also including the microscopic effects of reactants. 


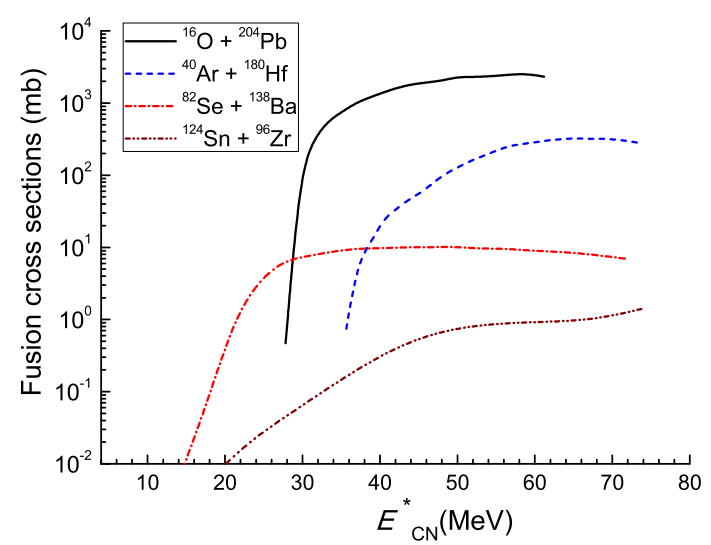

Figure 2. (Color online) As in Fig.1 but for the fusion excitation functions.

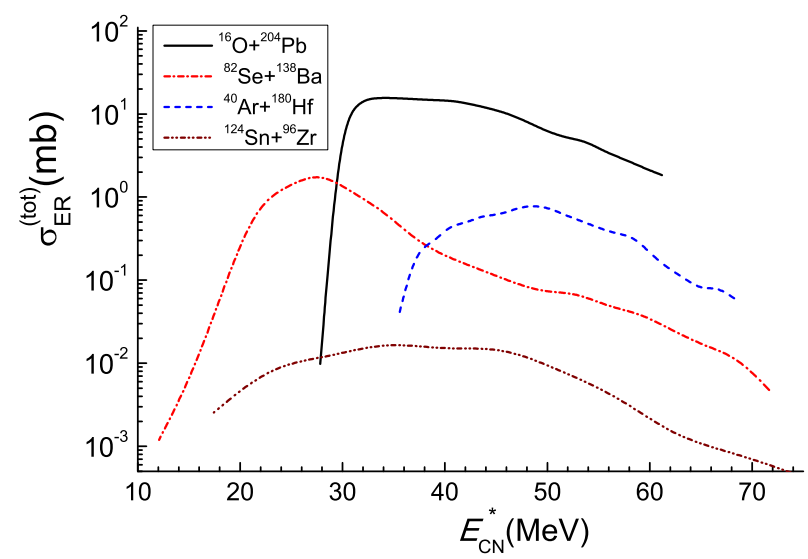

Figure 3. (Color online) As in Fig. 1 but for the total ER excitation functions.

It is useful to note that the threshold of the excitation energy of the $\mathrm{CN}$ formed by ${ }^{82} \mathrm{Se}+{ }^{138} \mathrm{Ba}$ (less mass symmetric reaction) is low as $12 \mathrm{MeV}$ while the one formed by ${ }^{40} \mathrm{Ar}+{ }^{180} \mathrm{Hf}$ (less mass asymmetric reaction) is higher as $37 \mathrm{MeV}$.

The influence of the entrance channel effects on the characteristics of the ER yields in the reactions leading to the same $\mathrm{CN}$ is studied by the comparison of the capture, fusion, and ER cross sections calculated by the combined dinuclear system and advanced statistical models $[12,14]$. The difference between ER cross sections can be related to the stage of formation of the $\mathrm{CN}$ or at its surviving stage against fission by emission of neutrons and charged particles. The comparison of theoretical results for the investigated reactions shows that the capture excitation functions obtained for the mass asymmetric reactions ${ }^{16} \mathrm{O}+{ }^{204} \mathrm{~Pb}$ and ${ }^{40} \mathrm{Ar}+{ }^{180} \mathrm{Hf}$ are one order of magnitude higher than the ones for the almost mass symmetric reaction ${ }^{82} \mathrm{Se}+{ }^{138} \mathrm{Ba}$ and ${ }^{124} \mathrm{Sn}+{ }^{96} \mathrm{Zr}$. The more strong Coulomb force makes the potential well shallower, and as a result the decrease of the number of partial waves $\left(\ell_{d}(E)\right)$ causes decreasing the capture cross section.
Comparison of the complete fusion results presented in Fig. 2 shows that the fusion excitation functions of the ${ }^{82} \mathrm{Se}+{ }^{138} \mathrm{Ba}$ and ${ }^{124} \mathrm{Sn}+{ }^{96} \mathrm{Zr}$ reactions are even two orders of magnitude lower than the ones of the mass asymmetric reactions. This result is explained by the hindrance to complete fusion due to the larger intrinsic fusion barrier $B_{\text {fus }}^{*}$ for the transformation of the DNS into $\mathrm{CN}$ and the smaller quasifission barrier $B_{\mathrm{qf}}$ in comparison with the corresponding quantities for the more asymmetric reactions. According to our calculations $B_{\text {fus }}^{*}$ increases and $B_{\mathrm{qf}}$ decreases by the increase of the DNS angular momentum.

The ER cross sections at the given values of the $\mathrm{CN}$ excitation energy $E_{\mathrm{CN}}^{*}$ and angular momentum $l$ are calculated in the framework of the advanced statistical model by the use of the partial cross sections of the $\mathrm{CN}$ formation obtained in the DNS model. The comparison of the theoretical excitation functions of the $x n$ evaporation residues formed in the ${ }^{16} \mathrm{O}+{ }^{204} \mathrm{~Pb},{ }^{40} \mathrm{Ar}+{ }^{180} \mathrm{Hf},{ }^{82} \mathrm{Se}+{ }^{138} \mathrm{Ba}$, and ${ }^{124} \mathrm{Sn}+{ }^{96} \mathrm{Zr}$ reactions (see Fig. 3) shows that the ER yields of the ${ }^{16} \mathrm{O}+{ }^{204} \mathrm{~Pb}$ is larger than the ones of the other three reactions since the fusion excitation function of this reaction is highest among the others. There is no hindrance to complete fusion for the reaction induced by the ${ }^{16} \mathrm{O}$ beam.

The ER- $x n$ and total ER excitation functions for the almost symmetric ${ }^{82} \mathrm{Se}+{ }^{138} \mathrm{Ba}$ reaction are about one and two orders of magnitude, respectively, higher than the ones of the other similar ${ }^{124} \mathrm{Sn}+{ }^{96} \mathrm{Zr}$ reaction. This is explained by the fact that the fusion excitation function of the former reaction is higher than the one of the latter reaction.

The unusual prevalence of the ${ }^{82} \mathrm{Se}+{ }^{138} \mathrm{Ba}$ reaction for the ER yields in the range $E_{C N}^{*}<38 \mathrm{MeV}$ in comparison with the mass asymmetric ${ }^{40} \mathrm{Ar}+{ }^{180} \mathrm{Hf}$ reaction which has larger fusion excitation function at $E_{C N}^{*}>38 \mathrm{MeV}$ is analyzed. The main significant result is related to the fact that the maximum value of the ER excitation function for the ${ }^{82} \mathrm{Se}+{ }^{138} \mathrm{Ba}$ reaction is sufficiently larger than the one for the ${ }^{40} \mathrm{Ar}+{ }^{180} \mathrm{Hf}$ reaction. In the $E_{C N}^{*}<35 \mathrm{MeV}$ energy range the fusion induced by ${ }^{40} \mathrm{Ar}$ is strongly hindered by the Coulomb barrier of the entrance channel. Therefore, due to relatively small $Q_{g g}$ value $(-99.49 \mathrm{MeV})$ for this reaction the lowest value of the excitation energy is $E_{C N}^{*}=35 \mathrm{MeV}$. Nevertheless, for the $E_{C N}^{*}>38 \mathrm{MeV}$ energy range the total ER yields produced by the ${ }^{40} \mathrm{Ar}+{ }^{180} \mathrm{Hf}$ reaction are higher than the ones produced by the ${ }^{82} \mathrm{Se}$ induced reaction due to the strong increase of the fusion cross section of the former reaction. The total ER yields decrease with the increase of the $E_{\mathrm{CN}}^{*}$ energy because at large excitation energy values this revealed trend is common to all reactions since the survival probability $W_{\text {sur }}$ decreases with the increase of the fission probability [13]. At the large beam energies the number of the partial waves contributing to fusion increases causing the decrease of the fission barrier $[15,16]$. The presence of the contribution of large values of $L$ is confirmed by the larger values of the total ER cross sections measured in the experiment [17]. The larger values of the total ER cross sections indicate the large contribution of the charged (proton and alpha) particles emission by $\mathrm{CN}$ during its de-excitation in the $E_{C N}^{*}>38 \mathrm{MeV}$ range. 


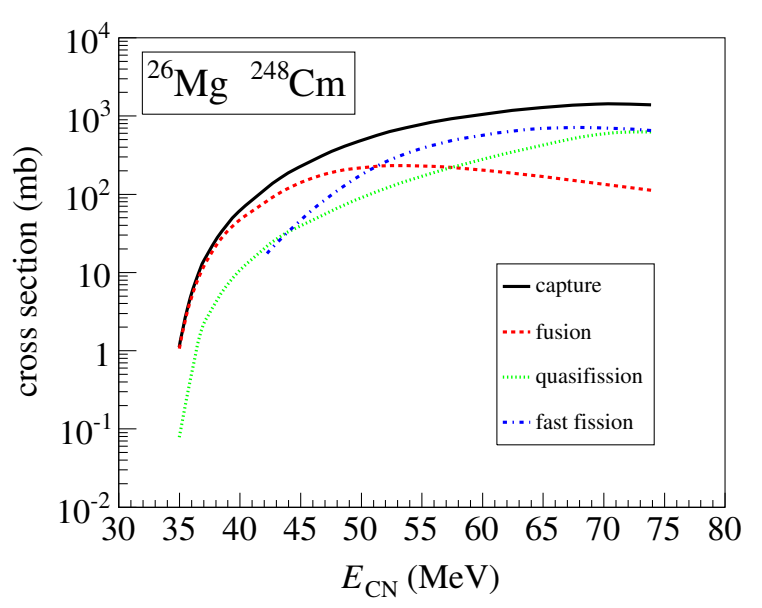

Figure 4. (Color online) Comparison of the capture, fusion, quasifission and fast fission excitation functions calculated for the ${ }^{26} \mathrm{Mg}+{ }^{248} \mathrm{Cm}$ reaction leading to the ${ }^{274} \mathrm{Hs}^{*} \mathrm{CN}$.

The comparison between the results of the ${ }^{40} \mathrm{Ar}+{ }^{180} \mathrm{Hf}$ and ${ }^{82} \mathrm{Se}+{ }^{138} \mathrm{Ba}$ reactions shows that the fusion probability $P_{\mathrm{CN}}$ and the survival probability $W_{\text {sur }}$ are important quantities characterizing different stages of the reaction during the formation of the $\mathrm{CN}$ and its surviving against fission.

The theoretical analysis of the measured data of the total ER yields by the combined the DNS and advanced statistical models allowed us to reveal the important role of the angular momentum distribution of the capture, complete fusion, and de-excitation stages of the mass asymmetric and mass symmetric reactions [13].

\subsection{Analysis of the ${ }^{26} \mathrm{Mg}+{ }^{248} \mathrm{Cm}$ and ${ }^{36} \mathrm{~S}+{ }^{238} \mathrm{U}$ reactions.}

These reactions lead to the same ${ }^{274} \mathrm{Hs}$ superheavy $\mathrm{CN}$ with $Z=108$ and $A=274$, and they are characterized by the mass asymmetry parameter values of 0.81 and 0.74 , respectively. The asymmetry parameters of these two reactions are a little different, but both reactions are clear mass asymmetric reactions.

In Figs. 4 and 5 we present the capture, fusion, quasifission, and fast fission excitation functions for the ${ }^{26} \mathrm{Mg}+{ }^{248} \mathrm{Cm}$ and ${ }^{36} \mathrm{~S}+{ }^{238} \mathrm{U}$ reactions, respectively.

The capture excitation functions obtained for the more mass asymmetric ${ }^{26} \mathrm{Mg}+{ }^{248} \mathrm{Cm}$ reaction is about a factor 2 higher than the ones for the less mass asymmetric ${ }^{36} \mathrm{~S}+{ }^{238} \mathrm{U}$ reaction. This difference in the capture cross section is related with the size of the potential well in the nucleus-nucleus interaction. Since the Coulomb repulsion is stronger for the less asymmetric reaction in comparison with the one for the more asymmetric reaction then will be $\left(Z_{1} \cdot Z_{2}\right)_{\mathrm{Mg}+\mathrm{Cm}}<\left(Z_{1} \cdot Z_{2}\right)_{\mathrm{S}+\mathrm{U}}$. The strong repulsion forces makes the potential well shallower, reducing consequently the maximum number of the partial waves $\left(\ell_{d}(E)\right)$ leading to capture, and then the capture cross section decreases. The difference between fusion excitation functions for the two considered reactions is explained by the hindrance to

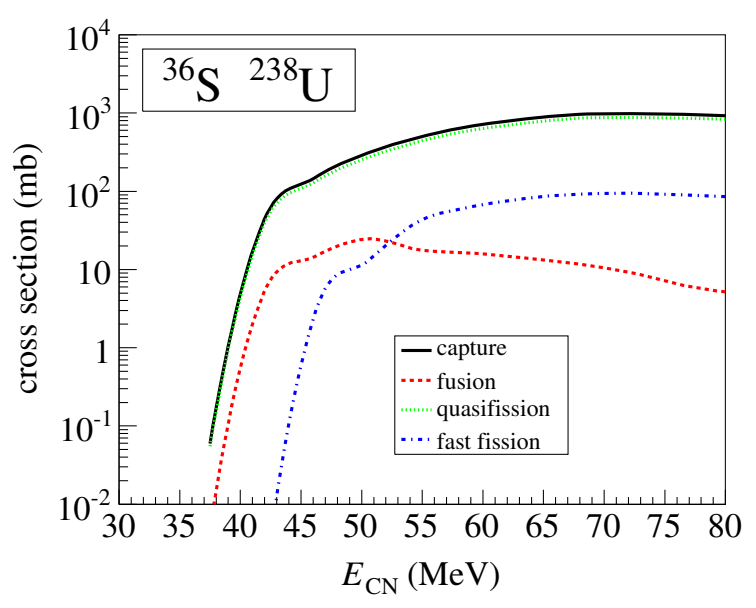

Figure 5. (Color online) As Fig. 4 but for the ${ }^{36} \mathrm{~S}+{ }^{238} \mathrm{U}$ reaction.

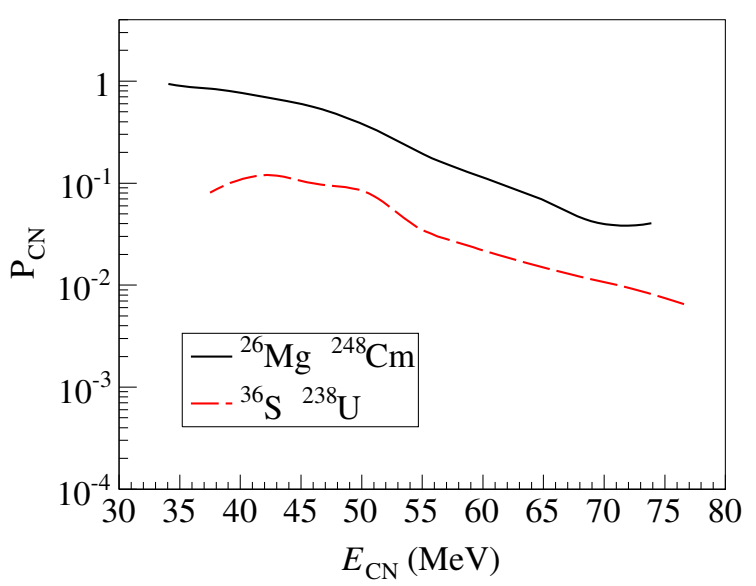

Figure 6. (Color online) Comparison of the fusion probability values for the two ${ }^{26} \mathrm{Mg}+{ }^{248} \mathrm{Cm}$ and ${ }^{36} \mathrm{~S}+{ }^{238} \mathrm{U}$ reactions.

complete fusion due to the larger intrinsic fusion barrier $B_{\text {fuS }}^{*}$ for the transformation of the DNS into a $\mathrm{CN}$ for the ${ }^{36} \mathrm{~S}+{ }^{238} \mathrm{U}$ reaction and the increase of the quasifission contribution due to the decreasing of the quasifission barrier $B_{\mathrm{qf}}$ as a function of the angular momentum. Fig. 6 shows comparison of the fusion probability values vs the $E_{\mathrm{CN}}^{*}$ excitation energy for the two reactions leading to the same ${ }^{274} \mathrm{Hs} \mathrm{CN}$. It is seen that the largest $P_{\mathrm{CN}}$ values obtained for the more mass asymmetric ${ }^{26} \mathrm{Mg}+{ }^{248} \mathrm{Cm}$ reaction is larger than the ones of the ${ }^{36} \mathrm{~S}+{ }^{238} \mathrm{U}$ reaction.

Since $V_{B}$ depends on the distance $R$ between the centers of the two reacting nuclei at the time of their contact and also on the angular configurations of the two axial symmetry axes of nuclei $i$ which are allowed to form DNS events (and then probably to reach the $\mathrm{CN}$ formation), it is clear that the $E_{\text {c.m. }}$ beam energies enough to give contributes to the partial capture cross section are influenced on the dynamic of nuclei in the entrance channel. Of course this kind of sensitivity is relevant for beam ener- 
gies lower than the conventional interaction barrier, and it also involves the $P_{\mathrm{CN}}$ probability to reach the $\mathrm{CN}$ formation to cause of the $B_{\text {fus }}^{*}$ and $B_{\mathrm{qf}}$ barrier values which are sensitive to the entrance channel dynamics $[8,14,18]$. In addition, it useful to note that at the de-excitation of $\mathrm{CN}$, reached also at the same $E_{\mathrm{CN}}^{*}$ excitation energy by two different entrance channel reactions, the excitation functions of reaction products are strongly sensitive to the entrance channel dynamics $[8,18]$.

\subsection{Comparison of $P_{\mathrm{CN}}$ fusion probabilities for the ${ }^{48} \mathrm{Ca}+{ }^{144,154} \mathrm{Sm}$ and ${ }^{16} \mathrm{O}+{ }^{186} \mathbf{W}$ reactions.}

The ${ }^{48} \mathrm{Ca}+{ }^{144,154} \mathrm{Sm}$ reactions induced by the ${ }^{48} \mathrm{Ca}$ beam on the two different neutron rich ${ }^{144,154} \mathrm{Sm}$ targets lead to the two ${ }^{192,202} \mathrm{~Pb} \mathrm{CNs}$, isotopes of the lead. The ${ }^{48} \mathrm{Ca}$ beam is a double shell-closure nucleus; the ${ }^{144} \mathrm{Sm}$ is a neutron shell-closure nucleus; the ${ }^{154} \mathrm{Sm}$ target is an enough neutron rich nucleus; the ${ }^{192,202} \mathrm{~Pb}$ CNs are both proton shellclosure nuclei. The ${ }^{16} \mathrm{O}+{ }^{186} \mathrm{~W}$ reaction leads to the ${ }^{202} \mathrm{~Pb}$ $\mathrm{CN}$ (a proton shell-closure nucleus) and it is characterized by a double shell-closure beam nucleus and an enough neutron rich target nucleus. In this last case the ${ }^{202} \mathrm{~Pb}$ $\mathrm{CN}$ is reached by a mass asymmetric reaction in comparison with the ${ }^{48} \mathrm{Ca}+{ }^{154} \mathrm{Sm}$ reaction that reaches the same ${ }^{202} \mathrm{~Pb} \mathrm{CN}$ by a relatively more symmetric one. We select these reactions in order to compare the results of the fusion probability and to show the sensitivity of our model with respect to the characteristics of the various reactions and to demonstrate the necessity of taking into account in calculations the shell structure and dynamical aspects of the entrance channel reactions. As examples we present in Fig. 7 (a) the fusion probability $P_{\mathrm{CN}}$ vs the angular momentum $l$ for the ${ }^{48} \mathrm{Ca}+{ }^{144,154} \mathrm{Sm}$ reaction at two different $E_{\mathrm{CN}}^{*}$ values of 49 and $63 \mathrm{MeV}$ and in Fig. 7 (b) the function $P_{\mathrm{CN}}$ vs the comparable effective energy $\left(E_{c . m .}-E_{B}\right)$ in the c.m. system for the ${ }^{16} \mathrm{O}+{ }^{186} \mathrm{~W},{ }^{48} \mathrm{Ca}+{ }^{144} \mathrm{Sm}$, and ${ }^{48} \mathrm{Ca}+{ }^{154} \mathrm{Sm}$ reactions.

As one can see, Fig. 7 (a) shows for the ${ }^{48} \mathrm{Ca}+{ }^{154} \mathrm{Sm}$ reaction relevant effect on the fusion probability values at each $\ell$-value when the excitation energy of $\mathrm{CN}$ changes from $49 \mathrm{MeV}$ (dashed line) to $63 \mathrm{MeV}$ (full line). Such an effect strongly changes the ratio of the $P_{\mathrm{CN}}$ values corresponding to these energies by factor 1.3 at low $\ell$-value up to factor 30 at higher $\ell$-values. Therefore, it is unlikely that determinations and model do not take into account the dynamic effects of the entrance channel on the $\mathrm{CN}$ formation and the following de-excitation cascade leading to the reaction products.

Figure 7 (b) shows the comparison of the $P_{\mathrm{CN}}$ values vs the $\left(E_{c . m .}-E_{B}\right)$ effective beam energy above the barrier of the nucleus-nucleus potential for the very mass asymmetric ${ }^{16} \mathrm{O}+{ }^{186} \mathrm{~W}$ reaction and the two close more symmetric ${ }^{48} \mathrm{Ca}+{ }^{144,154} \mathrm{Sm}$ reactions. Up to value $50 \mathrm{MeV}$ of the effective beam energy the ratio of the $P_{\mathrm{CN}}$ values for the two reactions with the ${ }^{144} \mathrm{Sm}$ and ${ }^{154} \mathrm{Sm}$ targets is not higher than by factor 1.5 with a $P_{\mathrm{CN}}$ maximum value of about 0.36 . The $P_{\mathrm{CN}}$ line of the slightly more mass symmetric ${ }^{48} \mathrm{Ca}+{ }^{144} \mathrm{Sm}$ reaction is a bit higher than the one for the
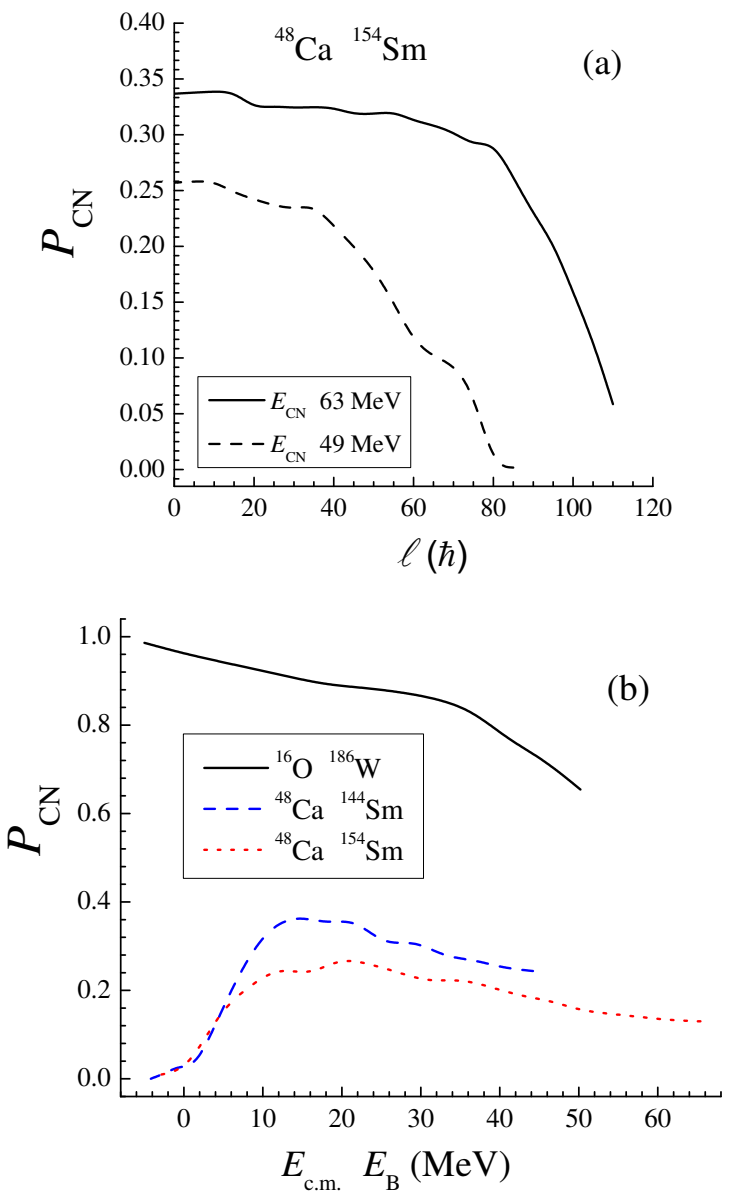

Figure 7. (Color online) (a) Fusion probability $P_{\mathrm{CN}}$ vs the angular momentum $l$ for the ${ }^{48} \mathrm{Ca}+{ }^{154} \mathrm{Sm}$ reaction at 49 and $63 \mathrm{MeV}$ of excitation energies. (b) $P_{\mathrm{CN}}$ values vs the $\left(E_{c, m}-E_{B}\right)$ effective beam energy overcoming the conventional interaction barrier $V_{B}$ for the ${ }^{16} \mathrm{O}+{ }^{186} \mathrm{~W}$ (very mass asymmetric reaction) and ${ }^{48} \mathrm{Ca}+{ }^{144,154} \mathrm{Sm}$ (more symmetric reactions) reactions.

more symmetric ${ }^{48} \mathrm{Ca}+{ }^{154} \mathrm{Sm}$ reaction. This result is also a little bit unexpected since often it is observed that reactions involving nuclei with more rich neutron show higher fusion cross section. In addition, Fig. 7 (b) also shows that the ${ }^{16} \mathrm{O}+{ }^{186} \mathrm{~W}$ very mass asymmetric reaction is characterized by a high $P_{\mathrm{CN}}$ fusion probability (about 1 ) at low energy, and the $P_{\mathrm{CN}}$ values slowly decrease with the increase of the beam energy. This effect reveals that also for very mass asymmetric reactions, at higher beam energies, the quasifission process competes with complete fusion by a relevant mode. This result was often not considered in heavy ion collisions induced by ${ }^{12} \mathrm{C},{ }^{14} \mathrm{~N},{ }^{16} \mathrm{O},{ }^{19} \mathrm{~F}$, and heavier beam nuclei.

\subsection{Very close reactions induced by ${ }^{26,25} \mathrm{Mg}$ on the ${ }^{248} \mathrm{Cm}$ target}

In the present Subsection we would like to study the appreciable effects that appear on the intermediate excited nuclei and evaporation residue nuclei formed along the deexcitation cascade of the two close compound nuclei (different only for 1 neutron) reached in the ${ }^{26,25} \mathrm{Mg}+{ }^{248} \mathrm{Cm}$ 


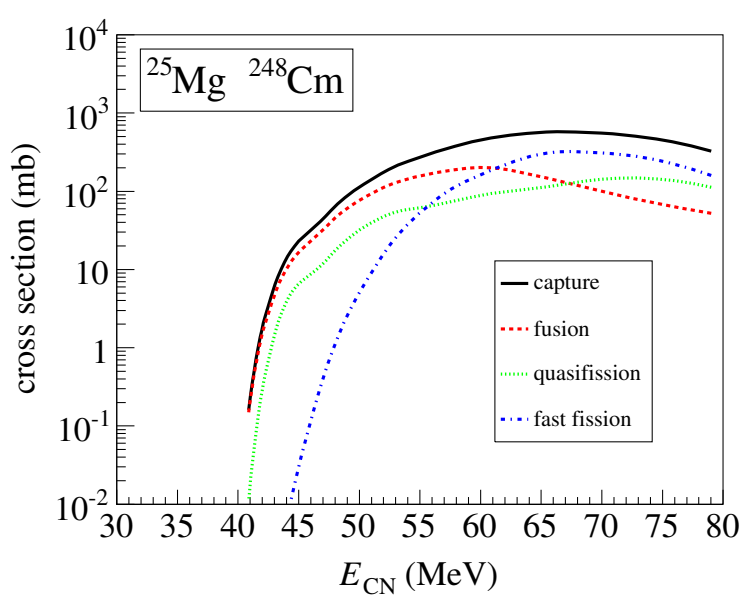

Figure 8. (Color online). As Fig. 4 but for the ${ }^{25} \mathrm{Mg}+{ }^{248} \mathrm{Cm}$ reaction leading to the ${ }^{273} \mathrm{Hs} \mathrm{CN}$.

reactions. We choose this kind of reactions leading to the ${ }^{274,273} \mathrm{Hs}$ compound nuclei, which widely investigated also by the other reactions, since there are available the experimental results in literature regarding the ER nuclei formed after neutron emission only from the hassium CN [19]. In Fig. 4 are presented the capture, fusion, quasifission, and fast fission excitation functions vs $E_{C N}^{*}$ for the ${ }^{26} \mathrm{Mg}+{ }^{248} \mathrm{Cm}$ reaction leading to the ${ }^{274} \mathrm{Hs}^{*} \mathrm{CN}$. In Fig. 8 the same kind of cross sections, as in Fig. 4 but only for the ${ }^{25} \mathrm{Mg}+{ }^{248} \mathrm{Cm}$ reaction leading to the ${ }^{273} \mathrm{Hs}^{*} \mathrm{CN}$, are presented.

In the case of the ${ }^{26} \mathrm{Mg}$ induced reaction we obtain the capture cross section with the maximum value of $1427 \mathrm{mb}$, the fusion cross section with the maximum value of 229 $\mathrm{mb}$, the quasifission cross section with the maximum of $624 \mathrm{mb}$, the maximum value of the fast fission cross section of about $709 \mathrm{mb}$, and the ER cross section after neutron emission only at $E_{C N}^{*}=63 \mathrm{MeV}$ of about $25 \mathrm{pb}$ (in acceptable agreement with the data of [19]). The ratio between the ER and fusion yields is about $1.4 \times 10^{-10}$ for the reaction induced by ${ }^{26} \mathrm{Mg}$. In the case of the ${ }^{25} \mathrm{Mg}$ induced reaction we obtain the maximum value of $\sigma_{c a p}$ of about $569 \mathrm{mb}$, the maximum value of $\sigma_{f u s}$ of about $200 \mathrm{mb}$, the maximum value of $\sigma_{q f i s}$ of about $144 \mathrm{mb}$, the maximum value of the fast fission of about $311 \mathrm{mb}$; the maximum value of $\sigma_{E R}$ (after neutron emission only) at $E_{C N}^{*}=63$ $\mathrm{MeV}$ is about $0.5 \mathrm{pb}$ (in agreement with the result of [20]).

Comparison of Figs. 4 and 8 show that the capture excitation function (full line) of the ${ }^{26} \mathrm{Mg}+{ }^{248} \mathrm{Cm}$ reaction is much higher than the one (full line) of the ${ }^{25} \mathrm{Mg}+{ }^{248} \mathrm{Cm}$ reaction. The threshold energy for the former reaction is about $35 \mathrm{MeV}$ while the one for the latter reaction is about $41 \mathrm{MeV}$. Also in this case we observe that, differently from the general rule, the less mass asymmetric reaction (induced by ${ }^{25} \mathrm{Mg}$ ) has a threshold energy higher than the one corresponding to the more asymmetric reaction (induced by ${ }^{26} \mathrm{Mg}$ ). Such a result is due to the different entrance channel dynamics for the two reactions. In fact, the ${ }^{26} \mathrm{Mg}$ has a large oblate deformation in the

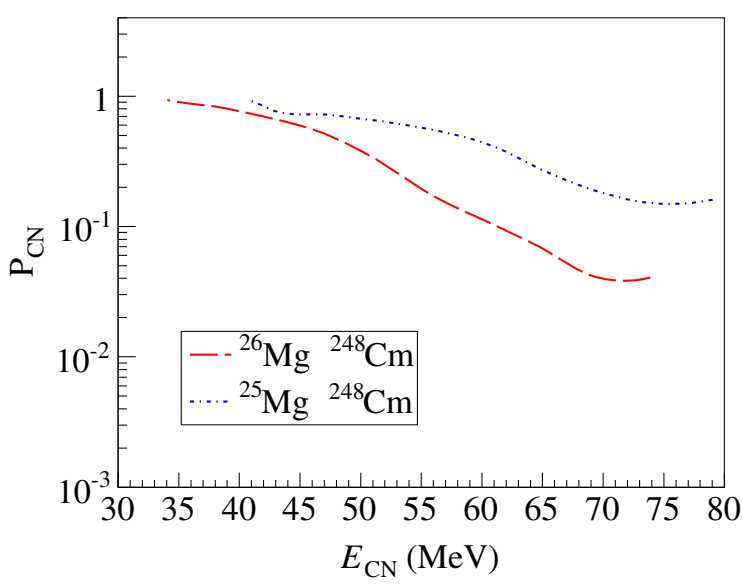

Figure 9. (Color online) Comparison of the fusion probability values for the two ${ }^{25} \mathrm{Mg}+{ }^{248} \mathrm{Cm}$ and ${ }^{26} \mathrm{Mg}+{ }^{248} \mathrm{Cm}$ reactions.

ground state $\left(\beta_{2}=-0.309\right.$ and $\left.\beta_{4}=-0.065\right)$ while ${ }^{25} \mathrm{Mg}$ is a strongly prolate deformed nucleus $\left(\beta_{2}=0.363\right.$ and $\beta_{4}=0.075$ ). Such a big difference in deformation of the ${ }^{25,26} \mathrm{Mg}$ beams leads to a large difference in the nucleusnucleus potential and in the angular momentum spectrum in the entrance channel, starting from the capture stage up to the ${ }^{273,274} \mathrm{Hs}^{*}$ compound nuclei formation. The dynamics of the entrance channel determines the evolution of the DNS and then the quasifission-fusion competition. Therefore, such dynamic characteristics of the ${ }^{274} \mathrm{Hs}^{*}$ and ${ }^{273} \mathrm{Hs}^{*}$ $\mathrm{CN}$ as spin distribution are different. A fortiori, the ${ }^{273} \mathrm{Hs}^{*}$ intermediate excited nucleus formed after 1 neutron emission from the ${ }^{274} \mathrm{Hs}^{*} \mathrm{CN}$ is dynamically different from the ${ }^{273} \mathrm{Hs}^{*} \mathrm{CN}$ formed in the reaction induced by the ${ }^{25} \mathrm{Mg}$ beam, even if in this last case it is reached at the same excitation energy. The same considerations are valid also for the other intermediate excited nuclei ${ }^{272} \mathrm{Hs}^{*},{ }^{271} \mathrm{Hs}^{*}$ and ${ }^{270} \mathrm{Hs}^{*}$ formed along the de-excitation cascades of the two above-mentioned reactions. Moreover, the maximum values of the ratios between $\sigma_{f u s}$ and $\sigma_{c a p}$ are about 0.16 and 0.35 for the ${ }^{26} \mathrm{Mg}$ and ${ }^{25} \mathrm{Mg}$ induced reactions, respectively. Therefore, the behavior of the competition between quasifission and complete fusion processes during the evolution of DNS is very different for the two studied reactions. The comparison of the $P_{\mathrm{CN}}$ fusion probabilities vs $E_{C N}^{*}$ for the two above-mentioned reactions is presented in Fig. 9.

Analogously, the fission process competes with the evaporation of light particles at decay of $\mathrm{CN}$, and the decay properties of the following intermediate excited nuclei reached along the de-excitation cascade of $\mathrm{CN}$ are very different for the two considered reactions. The $\sigma_{\mathrm{ER}} / \sigma_{\text {fus }}$ ratio is about $10^{-10}$ and $10^{-12}$ for the ${ }^{26} \mathrm{Mg}+{ }^{248} \mathrm{Cm}$ and ${ }^{25} \mathrm{Mg}+{ }^{248} \mathrm{Cm}$ reactions, respectively, at $E_{C N}^{*}=63 \mathrm{MeV}$. Moreover, Fig. 10 shows the $\Gamma_{\mathrm{n}} / \Gamma_{\text {tot }}$ ratios for the excited nuclei along the de-excitation cascade vs the $E_{\mathrm{CN}}^{*}$ excitation energy of the ${ }^{274} \mathrm{Hs}^{*} \mathrm{CN}$ formed in the reaction induced by ${ }^{26} \mathrm{Mg}$. Fig. 11 shows the similar $\Gamma_{\mathrm{n}} / \Gamma_{\text {tot }}$ ratios along the cascade vs the $E_{\mathrm{CN}}^{*}$ excitation energy of the 


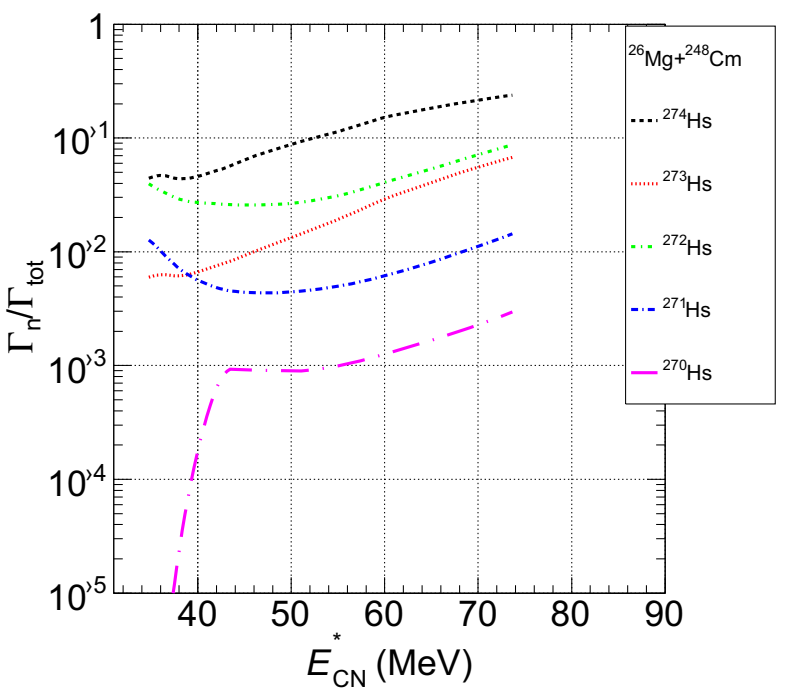

Figure 10. (Color online). The $\Gamma_{\mathrm{n}} / \Gamma_{\text {tot }}$ values for the excited nuclei along the de-excitation cascade vs the $E_{\mathrm{CN}}^{*}$ excitation energy of the ${ }^{274} \mathrm{Hs}^{*} \mathrm{CN}$ formed in the ${ }^{26} \mathrm{Mg}+{ }^{248} \mathrm{Cm}$ reaction.

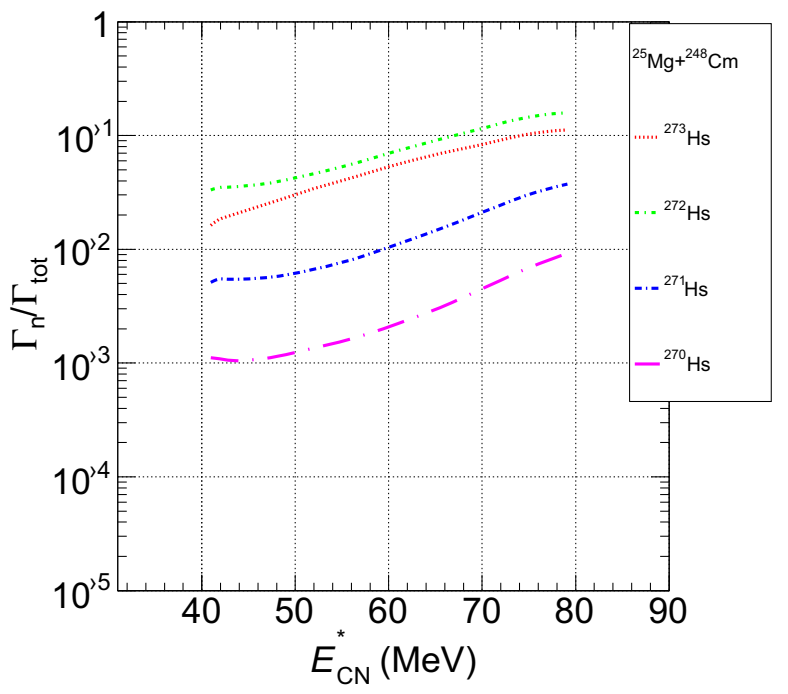

Figure 11. (Color online). As Fig. 10 but for the ${ }^{273} \mathrm{Hs}^{*} \mathrm{CN}$ formed in the ${ }^{25} \mathrm{Mg}+{ }^{248} \mathrm{Cm}$ reaction.

${ }^{273} \mathrm{Hs}^{*} \mathrm{CN}$ formed in the reaction induced by ${ }^{25} \mathrm{Mg}$ on the same ${ }^{248} \mathrm{Cm}$ target.

Figs. 10 and 11 show the $\Gamma_{\mathrm{n}} / \Gamma_{\text {tot }}$ values range for the two investigated reactions for each intermediate excited nucleus reached along the cascade as a function of $E_{\mathrm{CN}}^{*}$. These results are obtained by assuming that at each neutron emission the excited nucleus losses $10 \mathrm{MeV}$, and it becomes an intermediate excited nucleus; and so on at each step of the cascade. Figs. 10 and 11 provide us the following information: if, for example, the ${ }^{274} \mathrm{Hs}^{*} \mathrm{CN}$ formed in the ${ }^{26} \mathrm{Mg}+{ }^{248} \mathrm{Cm}$ reaction at the excitation en$\operatorname{ergy} E_{C N}^{*}=74 \mathrm{MeV}$, emits 1 neutron, it becomes the
${ }^{273} \mathrm{Hs}^{*}$ intermediate nucleus having an average excitation energy of about $64 \mathrm{MeV}$ with a relevant energy spread caused by the kinetic energy spectrum of the neutron emission; moreover, after the emission of 1 neutron the ${ }^{273} \mathrm{Hs}^{*}$ intermediate nucleus achieves the ${ }^{273} \mathrm{Hs}^{*}$ excited intermediate nucleus with an average excitation energy of about $54 \mathrm{MeV}$ and a wider energy spread; and so on at each step of the cascade. As it is seen from Fig. 10 , the $\Gamma_{n} / \Gamma_{\text {tot }}$ ratio is about 0.24 for the emission of the first neutron from the ${ }^{274} \mathrm{Hs}^{*} \mathrm{CN}$ at $E_{C N}^{*}=74 \mathrm{MeV}$. In the following step, the emission of $1 \mathrm{n}$ from ${ }^{273} \mathrm{Hs}^{*}$ at $64 \mathrm{MeV}$ of excitation energy corresponds to the $\Gamma_{\mathrm{n}} / \Gamma_{\text {tot }}$ ratio of about 0.038 . Instead, the $\Gamma_{\mathrm{n}} / \Gamma_{\text {tot }}$ ratio is about 0.066 for the emission of 1 neutron by the ${ }^{273} \mathrm{Hs}^{*} \mathrm{CN}$ reached by the ${ }^{25} \mathrm{Mg}+{ }^{248} \mathrm{Cm}$ reaction with the excitation energy of $E_{C N}^{*}=64 \mathrm{MeV}$. This value is about 1.74 times larger than the one obtained by the emission of $1 \mathrm{n}$ from ${ }^{273} \mathrm{Hs}^{*}$ formed after the emission of $1 \mathrm{n}$ from the ${ }^{274} \mathrm{Hs}^{*} \mathrm{CN}$. Moreover, the values of the $\Gamma_{\mathrm{n}} / \Gamma_{\text {tot }}$ ratio for the formation of the ${ }^{272} \mathrm{Hs}^{*}$ excited nucleus at $E^{*}=54 \mathrm{MeV}$ after the emission of 2 neutrons from the de-excitation cascade of the ${ }^{274} \mathrm{Hs}^{*} \mathrm{CN}$ (formed in the reaction induced by ${ }^{26} \mathrm{Mg}$ ) and after the emission of 1 neutron from the ${ }^{273} \mathrm{Hs}^{*} \mathrm{CN}$ (formed in the reaction induced by ${ }^{25} \mathrm{Mg}$ ) are equal to 0.030 and 0.051 , respectively. Also in this case we found for the $\Gamma_{\mathrm{n}} / \Gamma_{\text {tot }}$ ratios for the two mentioned reactions the value 1.7 times higher than the one obtained for the reaction induced by ${ }^{25} \mathrm{Mg}$.

We also obtain a ratio of about 1.25 between the $\Gamma_{\mathrm{n}} / \Gamma_{\text {tot }}$ values found in the next step of the de-excitation cascade, forming the ${ }^{271} H s^{*}$ excited nucleus at $E^{*}=44$ $\mathrm{MeV}$ after $3 \mathrm{n}$ emission from the ${ }^{274} \mathrm{Hs}^{*} \mathrm{CN}$, in comparison with $2 \mathrm{n}$ emission from the ${ }^{273} \mathrm{Hs}^{*} \mathrm{CN}$. Moreover, we obtain a larger ratio between the $\Gamma_{\mathrm{n}} / \Gamma_{\text {tot }}$ values vs $E_{\mathrm{CN}}^{*}$ calculated for the intermediate ${ }^{270} \mathrm{Hs}$ excited nucleus reached after 4 neutron emission from the ${ }^{274} \mathrm{Hs}^{*} \mathrm{CN}$, in comparison to the one obtained after 3 neutron emission from the ${ }^{273} \mathrm{Hs}^{*} \mathrm{CN}$.

Therefore, it is unlikely to think and assume that the excited nuclei as ${ }^{273} \mathrm{Hs}^{*},{ }^{272} \mathrm{Hs}^{*},{ }^{271} \mathrm{Hs}^{*},{ }^{270} \mathrm{Hs}^{*}$, etc. formed at comparable excited energies along the de-excitation cascade of two close reactions like ${ }^{26,25} \mathrm{Mg}+{ }^{248} \mathrm{Cm}$ give the same $\Gamma_{\mathrm{n}} / \Gamma_{\text {tot }}$ neutron emission probability at each step of the two considered cascades. We note that the authors of Ref. [21] found for the ${ }^{26} \mathrm{Mg}$ $+{ }^{248} \mathrm{Cm}$ reaction the $\Gamma_{\mathrm{n}} / \Gamma_{\text {tot }}$ value of $0.89 \pm 0.13$ for the emission of $1 \mathrm{n}$ from the ${ }^{274} \mathrm{Hs}^{*} \mathrm{CN}$ at $E_{C N}^{*}=63 \mathrm{MeV}$. As Fig. 10 shows, at about $E_{C N}^{*}=63 \mathrm{MeV}$ we obtain for the $\Gamma_{\mathrm{n}} / \Gamma_{\text {tot }}$ neutron emission probability the value of 0.17 , while the result presented in [21] is about 5.2 times higher.

Without going into the merits of the procedures and assumptions made by the authors of [21] during the experiment and data analysis through which the authors have come to determine the probability of the neutron emission $\Gamma_{\mathrm{n}} / \Gamma_{\text {tot }}$ equal to about 0.90 from the first step of the deexcitation cascade of the ${ }^{274} \mathrm{Hs}^{*} \mathrm{CN}$, through the observation of the neutron multiplicity, we believe it is necessary to make some comments and considerations.

At any value of the excitation energy $E_{\mathrm{CN}}^{*}$ of $\mathrm{CN}$, the emission of $1 \mathrm{n}$ from a rotating and excited nucleus does 
not appreciably change the range of the angular momentum distribution for not more than 1-2 $\hbar$ of the values of $\mathrm{CN}$; then, the reached intermediate excited nucleus is characterized by an angular momentum distribution similar to that of the $\mathrm{CN}$ but with a very different yields of the angular momentum distribution due to the limited neutron emission probability in order to reach the next intermediate excited nucleus with an excitation energy $10 \mathrm{MeV}$ lower. In the case of the ${ }^{26} \mathrm{Mg}+{ }^{248} \mathrm{Cm}$ reaction studied in [21], the ${ }^{274} \mathrm{Hs}^{*} \mathrm{CN}$ was achieved with an excitation energy $E_{\mathrm{CN}}^{*}$ of about $63 \mathrm{MeV}$. By considering that for the ${ }^{274} \mathrm{Hs}^{*}$ nucleus the macroscopic fission barrier is zero and therefore only the shell correction can help to provide an actual fission barrier different from zero; by observing also the tables $[22,23]$ where the shell corrections (and then the fission barriers) increase more than $1 \mathrm{MeV}$ passing from the ${ }^{274} \mathrm{Hs}$ nucleus to ${ }^{271} \mathrm{Hs}$, all these considerations lead to assert that there is a growth of the actual fission barrier for the intermediate nuclei along the de-excitation cascade of $\mathrm{CN}$. Then, the fission probability decreases and the ER nuclei should grow from the first chance to other following chances along the de-excitation cascade of the ${ }^{274} \mathrm{Hs}^{*} \mathrm{CN}$ and the intermediate excited nuclei.

If the found $\Gamma_{\mathrm{n}} / \Gamma_{\text {tot }}$ value is for example 0.9 at decay of the ${ }^{274} \mathrm{Hs}^{*} \mathrm{CN}$, then the $\Gamma_{\mathrm{n}} / \Gamma_{\text {tot }}$ values at decay of ${ }^{273} \mathrm{Hs}^{*}$, ${ }^{272} \mathrm{Hs}^{*}$ and other following nuclei will be higher than the $\Gamma_{\mathrm{f}} / \Gamma_{\text {tot }}$ values because the fission probabilities decreases with the increase of the fission barriers. Therefore, starting from the fusion cross section of about $200 \mathrm{mb}$ at $E_{C N}^{*}=$ $63 \mathrm{MeV}$, the total ER cross section should be of the order of some mb; instead, in the experiments $[19,20]$ the values found for the ER cross sections were of the order of $\mathrm{pb}$ (about 9 orders of magnitude lower). In our investigated ${ }^{25,26} \mathrm{Mg}$ induced reactions we obtain the maximum value of the total evaporation residue nuclei of the order of the tens of $\mathrm{pb}$ in the case of the reaction induced by ${ }^{26} \mathrm{Mg}$, and of the order of $\mathrm{pb}$ in the case of the reaction induced by ${ }^{25} \mathrm{Mg}$, in line with the experimental values. Therefore, the value of 0.9 of $\Gamma_{\mathrm{n}} / \Gamma_{\text {tot }}$ found in [21] at first step of one neutron emission from the ${ }^{274} \mathrm{Hs}^{*}$ excited nucleus at $E_{\mathrm{CN}}^{*}=63$ $\mathrm{MeV}$ appears incomprehensible in comparison with the value of 0.17 that we find (see Fig. 10). In our calculation we use the level density parameter $\mathrm{a}=\mathrm{A} / 8$, the masses of nuclei and the fission barrier taken from refs. [23, 24], and the damping functions of the fission barrier depending on the nuclear temperature and angular momentum $l$ as discussed in our previous papers (see for example ref. [12]).

\section{Conclusions}

The theoretical results discussed in the present paper, in comparison with the data obtained in four investigated reactions (from the more mass asymmetric ${ }^{16} \mathrm{O}+{ }^{204} \mathrm{~Pb}$ reaction to the almost mass symmetric ${ }^{124} \mathrm{Sn}+{ }^{96} \mathrm{Zr}$ reaction leading to the same ${ }^{220} \mathrm{Th} \mathrm{CN}$ ), allow us to affirm about the necessity to perform a detailed theoretical model in order to understand the significant differences that appear in the results of different investigated nuclear reactions.
The model should be able to describe a real varied evolution of the initial system formed by the two colliding nuclei - under the condition that it forms a pocket in the nucleus-nucleus potential- up to the formation of the final reaction products. In order to be able to correctly describe the full dynamic evolution of the reacting nuclear system, it is necessary that the model takes into account all the possible reaction mechanisms that can lead to the observed reaction products.

Obviously, to successfully reach this is not a simply final result from both theoretical and experimental point of view, it is necessary to know how to prepare a model that describes all the possible evolutions of the reacting nuclei in order to realistically calculate the various physical quantities (such as cross sections, mass distribution of the fragments and their angular distributions, energy distributions, characterization of the evaporation residue nuclei, etc.), through the relevant physical variables (nuclear deformation of reacting nuclei, mass asymmetry parameter, energy beam, geometric configuration of symmetry axes of nuclei at the capture stage) that determine the possible contributions to the reaction final products. At the same time, it is necessary that researchers are aware that some assumptions made in experiments on the limitation of the recorded events of the revealed products and further additional assumptions made in the procedure of analysis of the measured data, are not free from relevant ambiguities and uncertainties. Accordingly, the effects are not easily recognizable when one observes the experimental results. In fact, if one observes the data of the ER excitation functions for the above-mentioned four investigated reactions leading to ${ }^{220} \mathrm{Th}^{*} \mathrm{CN}$, it is very difficult to see, understand and explain some apparent anomalies or deviations from the standard rules.

For example, generally the fusion threshold $E_{\mathrm{CN}}^{*}$ values are lower for more mass symmetric reactions than the ones for the mass asymmetric reactions. Such a rule is verified for the mass asymmetric ${ }^{16} \mathrm{O}+{ }^{204} \mathrm{~Pb}$ and ${ }^{40} \mathrm{Ar}+{ }^{180} \mathrm{Hf}$ reactions in comparison with the mass symmetric ${ }^{82} \mathrm{Se}+{ }^{138} \mathrm{Ba}$ and ${ }^{124} \mathrm{Sn}+{ }^{96} \mathrm{Zr}$ reactions, but is not true for the ${ }^{40} \mathrm{Ar}+{ }^{180} \mathrm{Hf}$ (less asymmetric) in comparison with the ${ }^{16} \mathrm{O}+{ }^{204} \mathrm{~Pb}$ one (more asymmetric), and analogously for the ${ }^{124} \mathrm{Sn}+{ }^{96} \mathrm{Zr}$ (more symmetric) in comparison with the ${ }^{82} \mathrm{Se}+{ }^{138} \mathrm{Ba}$ reaction (less symmetric), as well as also for the very close ${ }^{26,25} \mathrm{Mg}$ induced reactions on the ${ }^{248} \mathrm{Cm}$ target, where the results of the fusion threshold $E_{\mathrm{CN}}^{*}$ values are inverted in comparison with the general rule. An analogous discrepancy appears when, for a fixed reaction, in some $E_{\mathrm{CN}}^{*}$ energy range the total ER yield increases with the increase of the $\mathrm{CN}$ excitation energy and, instead, in another following $E_{\mathrm{CN}}^{*}$ energy range the total ER yield decreases with the increase of the $\mathrm{CN}$ excitation energy.

Moreover, when in an experiment the fragments are detected as the final products of a reaction, and one seeks to identify the processes that lead to the formation of the final products only through the analysis of the characteristics and properties of the mass distribution of the fragments and/or their angular distribution, other difficulties and discrepancies can arise when one compares the ex- 
perimental results of the two close reactions. In fact, this for example occurs when one observes and compares the data of the ${ }^{26} \mathrm{Mg}+{ }^{248} \mathrm{Cm}$ and ${ }^{36} \mathrm{~S}+{ }^{238} \mathrm{U}$ reactions leading to the same ${ }^{274} \mathrm{Hs} \mathrm{CN}$, and also in the case of other two ${ }^{48} \mathrm{Ca}+{ }^{154} \mathrm{Sm}$ and ${ }^{48} \mathrm{Ca}+{ }^{144} \mathrm{Sm}$ reactions leading to different neutron rich isotopes of lead $\left({ }^{202} \mathrm{~Pb}\right.$ and ${ }^{192} \mathrm{~Pb}$, respectively). The main cause is due to the very different influence of the quasifission process that is in competition with the complete fusion. Therefore, without the availability of an appropriate and sensitive theoretical model that is able to describe the various processes that can be activated during the evolution of the system of reacting nuclei, it is very difficult to think that through a simply observation of experimental results it is possible to achieve an appropriate explanation of the complete reaction dynamics and the kinds of processes that determine the observed final products of the reaction.

These difficulties and uncertainties added to the not valuable results on data due to the inevitable assumptions made in the treatment of the recorded events, lead to a real difficulty of knowing the contributions of the various processes, the effects of which vary according to the type of reaction and to the beam energy range.

\section{Acknowledgements}

A.K. Nasirov thanks the MSIP of the Korean Government (Brain Pool Program No. 142S-1-3-1034).

\section{References}

[1] C.C. Sahm and H.-G. Clerc, K.-H. Schmidt, W. Reisdorf, P. Armbruster, F.P. Heberger, J.G. Keller, G. Münzenberg, and D. Vermeulen, Z.Phys. A 319, 113 (1984)

[2] D.J. Hinde, M. Dasgupta, and A. Mukherjee, Phys.Rev.Lett. 89, 282701, 2002.

[3] A.Yu. Chizhov, M.G. Itkis, I.M. Itkis, G.N. Kniajeva, E.M. Kozulin, N.A. Kondratiev, I.V. Pokrovsky, R.N. Sagaidak, V.M. Voskressensky, A.V. Yeremin, L. Corradi, A. Gadea, A. Latina, A.M. Stefanini, S. Szilner, M. Trotta, A.M. Vinodkumar, S. Beghini, G. Montagnoli, F. Scarlassara, A.Ya. Rusanov, F. Hanappe, O. Dorvaux, N. Rowley, and L. Stuttge, Phys. Rev. C67, 011603(R) (2003).

[4] Giovanni Fazio, Giorgio Giardina, Antonino Lamberto, Roberto Ruggeri, Carmelo Sacca, Rocco Palamara, Akhtam I. Muminov, Avazbek K. Nasirov, Ulughbek T. Yakhshiev, Francis Hanappe, Thomas Materna and Louise Stuttge, Jour. Phys. Soc. of Japan 72, 2509 (2003).
[5] M. Dasgupta and D.J. Hinde, Nucl. Phys. A734, 148 (2004).

[6] G. Fazio, G. Giardina, G. Mandaglio, and R. Ruggeri, A.I. Muminov, A.K. Nasirov, Yu.Ts. Oganessian, A.G. Popeko, R.N. Sagaidak, and A.V. Yeremin, S. Hofmann, F. Hanappe, C. Stodel, Phys. Rev. C 72, 064614 (2005).

[7] Avazbek Nasirov, Akira Fukushima, Yuka Toyoshima, Yoshihiro Aritomo, Akhtam Muminov, Shuhrat Kalandarov, Ravshanbek Utamuratov, Nucl. Phys. A759, 342 (2005).

[8] Giovanni Fazio, Giorgio Giardina, Francis Hanappe, Giuseppe Mandaglio, Marina Manganaro, Akhtam I. Muminov, Avazbek K. Nasirov, Carmelo Sacca, Jour. Phys. Soc. of Japan 77, 124201, (2008).

[9] G. Mandaglio, G. Fazio, G. Giardina, F. Hanappe, M. Manganaro, A.I. Muminov, A.K. Nasirov, and C. Sacca Physics of Atomic Nuclei, 2009, 72, 1639 (2009).

[10] G. Fazio, G. Giardina, G. Mandaglio, F. Hanappe, A.I. Muminov, A.K. Nasirov, W. Scheid, L. Stuttge, Mod. Phys. Lett. A 20, 391 (2005).

[11] B. B. Back, R. R. Betts, J. E. Gindler, B. D. Wilkins, S. Saini, M. B. Tsang, C. K. Gelbke, W. G. Lynch, M. A. McMahan, and P. A. Baisden, Phys. Rev. C 32, 195 (1985).

[12] G. Mandaglio, G. Giardina, A. K. Nasirov, and A. Sobiczewski, Phys. Rev. C 86, 064607 (2012).

[13] K. Kyungil, A.K. Nasirov et al., arXiv:1411.7239 [nucl-th], (2014).

[14] A. Nasirov et al., Eur. Phys. J. A 49, 147 (2013).

[15] G. Fazio, G. Giardina, A. Lamberto, A.I. Muminov, A.K. Nasirov, F. Hanappe, L. Stuttge, Eur. Phys. J. A 22, 75 (2004).

[16] H. Q. Zhang et al., Phys. Rev. C 81, 034611 (2010).

[17] D. Vermeulen, H.-G. Clerc, and C.C. Sahm, Z.Phys. A 318, 157 (1984).

[18] A. K. Nasirov, G. Giardina, G. Mandaglio, and M. Manganaro, F. Hanappe, S. Heinz and S. Hofmann, A. I. Muminov, W. Scheid, Phys. Rev. C 79, 024606 (2009).

[19] J. Dvorak et al., Phys. Rev. Lett. 100, 132503 (2008).

[20] J. Dvorak et al., Phys. Rev. C 79, 037602 (2009).

[21] R. Yanez et al., Phys. Rev. Lett. 112, 152702 (2014).

[22] P. Möller, J.R. Nix, W.D. Myers, and W.J. Swiatecki, Atomic Data and Nuclear Data Tables 59, 185 (1995).

[23] M.Kowal, P. Jachimowicz, and A. Sobiczewski, Phys. Rev. C 82, 014303 (2010).

[24] M.Kowal and A. Sobiczewski, Int. J. Mod. Phys. E 18, 914 (2009). 